It must be remembered that leprosy like tuberculosis is a bacillary disease, and unlike spirochætal diseases such as syphilis and yaws, does not readily respond to drugs. As in tuberculosis, so in leprosy, in the present state of our knowledge, we must depend as much if not more on segregation and a sanatorium régime to increase the resistance of the patient or on the use of protein shock in suitable cases than on any form of so-called specific therapy which has yet been introduced.

\title{
ROUND THE WARDS WITH MR. LOCKHART-MUMMERY AT ST. MARK'S HOSPITAL.
}

St. Mark's Hospital, in the City Road, London, is unequalled in the facilities it affords for studying a large number of cases of one particular region collected in a single building, and in charge of a staff of men who practise almost exclusively in this one particular branch of surgery. The Hospital contains seventy-five beds, and last year over I, roo operations were performed, all for conditions of the rectum or colon. There is at present no other institution in the world which affords similar opportunities of seeing many different kinds of disease, and abnormal conditions, of the large bowel. As regards equipment this Hospital is thoroughly up-to-date, and cases can be seen to the best advantage by anyone visiting the clinics.

\section{Case of Epithelioma of Anus Treated by Radium.}

The first case shown was that of a man, aged 55, who had originally come to the Hospital with an epithelioma of the anal margin. The case had been treated by radium nine months previously. The original tumour was about $\mathrm{I} \frac{1}{2}$ in. in diameter and extended out on to the skin of the perineum in front of the anus and up the anal canal for about an inch. Microscopic slides of the specimen removed for examination were demonstrated showing the typical appearance of squamous carcinoma. There were no enlarged glands. The case was treated with radon seeds and radium needles. Radium equivalent to $285 \mathrm{mg}$. was used and the total dose was about 2,500 mg. hours. The epitheliomatous ulcer began to heal in ten days and had entirely healed over in three and a half weeks. At the present time, nine months later, there is no sign of anything wrong. The anal opening is normal and there is no detectable scar.

It was pointed out that squamous-celled carcinoma could be very successfully treated with radium, and that if, as appeared to be the case in this instance, the correct dose was used the results were little short of miraculous. Unfortunately there was at the present time no accurate means of determining the proper dose, and the experience of the surgeon in similar cases was the only guide. It was pointed out that we are still quite in the dark as to the way in which radium acts upon the tumour. It was clearly not merely a question of the destruction of the cancer cells, as no sloughing occurred, the cancerous ulcer simply healed up, leaving normal tissue in its place. If, on the other hand, an overdose of radium was used either sloughing occurred or dense fibrous tissue was formed. But when the dose was correct the tissues simply resumed a normal appearance in the course of a few weeks without any violent reaction and with the minimum of scar tissue. 
Similar results had been obtained with adeno-carcinoma of the rectum, where the tumour was quite early and where it was quite low down on the posterior rectal wall, and therefore easily accessible for radium treatment and the correct placing of the needles or seeds. At the present day operation undoubtedly affords the best chance of curing patients suffering from cancer of the rectum, but the case shown made one hope that with improved technique we might in suitable cases be able to cure rectal cancer with radium without the necessity of having to remove the rectum by operation.

\section{Case of Cancer of the Rectum Treated by Operation.}

The next case was one of a man, aged 65, who had been operated on fourteen days previously for cancer of the rectum by perineal excision. The tumour was an adeno-carcinoma of the ampulla of the rectum, and involved two-thirds of the circumference. The operation had been performed under avertin and spinal anæsthesia, after a preliminary colostomy performed a week before. The patient was now getting up daily for a bath, and would be leaving the Hospital in about ten days time. The wound had healed, except for a small sinus in the perineum, in which there was a tube. It was pointed out that one of the great difficulties of these operations was to prevent infection of the bladder. This was largely due to the damage necessarily caused to the sympathetic nerve supply of the bladder resulting from removal of the rectum. It could be largely prevented by the use of a tied-in catheter attached to a Duke's apparatus, which was shown.

The prognosis in this case was fair, about a $5^{\circ}$ per cent. chance of complete immunity from recurrence. Cases at St. Mark's are all classified after the tumour hasô been examined in the pathological department. They are grouped as "A" cases if it is found that the cancer cells have not penetrated to the bowel wall, " $B$ " cases if they have penetrated, but have not invaded the lymphatics, and " $C$ " cases if the lymphatics are involved. This case was classed as a " $\mathrm{B}$ " case. The prognosis in an " $\mathrm{A}$ " case would be nearly roo per cent., and in a " $\mathrm{C}$ " case about 26 per cent.

The actual risk of the operation was now quite small, not more than 5 per cent., and the operation could be done on cases who were bad risks on account of other disease or age.

\section{Case of Congenital Deformity of the Anus.}

The next case was a small boy, aged 5 years. He was sent up to the Hospital because he had complete incontinence of fæces, a condition which had existed ever since he was born. He passed his stools into his clothes, and although he had been treated at the local hospital on several occasions there had been no improvement. On examination it could be seen that the boy had a congenital deformity of the anal opening. The opening was double, there being a band of skin passing antero-posteriorly across the anus and dividing it into two lateral openings. On the other hand there appeared to be a fairly normal opening above, and the sphincter muscle was present and closed the opening quite satisfactorily. It was pointed out that the deformity which existed did not seem to account for the loss of control over the stools.

The real cause of the incontinence was distension and overflow. Just as in the case 
of the bladder, over-distension results in overflow and incontinence of urine, so in the bowel the same causes could produce the same results. It was a well-known fact that old people, and patients recovering from serious illnesses, sometimes got incontinence, with constant diarrhœa, from over-distension of the rectum or colon with hard fæces. This child was a case in point, and since he had been completely emptied by means of enemas and castor oil he had obtained complete control over his evacuations, and was now, at any rate in that respect, a normal child. His parents, supposing that he was abnormal, had kept him constipated, with the result that the condition of incontinence had been produced from over-distension of the large bowel.

The other possible diagnosis in this case was megacolon, but the child had been $\mathrm{X}$-rayed after a barium enema, and films were shown which proved that there was no condition of megacolon present.

\section{Case of Congenital Absence of the Anus.}

The next case was one of a boy, aged 9, who had been born without any anal opening at all. He had been operated upon within twenty-four hours of birth, and an opening made into the bowel in the perineum. The opening was a very inadequate one, as for about $\frac{3}{4}$ in. it consisted of fibrous tissue, and had to be constantly dilated. This poor boy had constant pain when the bowels acted, and was in continual dread of having his anal opening dilated. It was proposed in this case to operate and form a proper opening with the mucous membrane down to the skin, and of sufficient size to prevent the necessity of constant dilatation. In these cases the bowel always takes an acute curve forwards and opens, often by a minute opening, into the bladder or prostatic urethra. (In the present case it was the latter.) By separating the bowel from the bladder or prostate, and straightening out the curve, sufficient bowel could be obtained to easily reach the skin in the normal situation of the anus.

\section{Case of Fistula.}

A case of semi-horseshoe fistula in a man who had been operated on ten days before, was next demonstrated. The track of the fistula had been completely cut out, except that where the track passed into the rectum above the external sphincter muscle it had been left, and a silk ligature placed round the muscle to act as a guide. Division of the sphincter at the original operation might have resulted in incontinence, but it was pointed out that by leaving the division until the remainder of the wound was almost healed this risk could be entirely avoided. The wound was seen to be healing well and to be very shallow. The silk ligature round the external sphincter could be seen and would act as a guide when in about another five days' time the sphincter was divided under local anæsthesia. It was stated that patients with fistula were not discharged from the hospital until quite healed up, and that the average time for healing was a few days over a month. Dressings were always changed twice daily after a bath and consisted of cotton-wool, not gauze, as wool resulted in less damage to the newly formed granulations. Antiseptics were not used after the first week for the same reason. 


\title{
Case of Carcinoma of the Colon treated by Resection.
}

The patient was a woman aged 56 . She came to the hospital with symptoms of increasing difficulty in getting the bowels to act, alternating constipation and diarrhœa, and occasional traces of blood in the stools. She also complained of a pain in the left iliac fossa, and a feeling that she wanted to relieve her bowels, without being able to get relief. An examination with the sigmoidoscope showed nothing beyond a little blood and mucus on the walls of the bowel high up-a rather significant finding. A barium enema showed a filling defect in the middle of the pelvic colon. This examination was repeated and the same appearance was found. It was pointed out how important it is to repeat X-ray examinations, as accidental appearances can be most misleading. The patient was kept in the hospital for a week, and her blood and blood urea tested; her colon thoroughly washed out and she was given glucose for two days prior to operation. The operation was performed under avertin anæsthesia and regional anæsthesia with 2 per cent. novocain. A diagonal incision was used and the tumour exposed and drawn up out of the wound. The bowel was divided above and below the tumour and the latter removed together with a wedge-shaped portion of the mesentery. The bowel was then joined by end to end union with catgut. The first suture taking up all the coats and the second the serous coat only. The omentum was stitched over the line of union to prevent the small gut becoming attached to it. A temporary tube cæcostomy was performed at the same time by the author's method.

This patient has made an excellent recovery and will be getting up in a few days. The temporary cæcostomy has now healed twelve days.

\section{INTRA-UTERINE GLYCERINE IN THE TREATMENT OF GONORRHAEA.}

\author{
By THOS. ANWYL-DAVIES, M.D., B.S.LOND., M.R.C.P.I.OND., \\ Director, L.C.C., Whitechapel Clinic, E. ; Consulting Venereologist to the London County Council ; \\ Lecturer in Venereal Diseases to the London Hospital Medical College, E.1.
}

Intra-Uterine Glycerine in the Treatment of Gonorrhœa.

Some twelve years ago my friend the late Remington Hobbs demonstrated to me the treatment of the septic uterus by glycerine irrigation, with the suggestion that his method might be useful in the treatment of gonorrhœa. This was so, and since then the advantages of drainage treatment over the application of caustics have been amply proved.

\section{Indications.}

Any signs or symptoms which lead one to suspect that gonococci have passed that natural anatomical barrier, the internal os, and invaded the uterus indicate that the introduction of glycerine into the uterine cavity should be carefully considered.

The gonorrhœal cases for which intra-uterine glycerine may be advisable can be 